\title{
Functional capacity of people with early osteoarthritis: a comparison between subjects from the cohort hip and cohort knee (CHECK) and healthy ageing workers
}

\author{
H. J. Bieleman · M. W. van Ittersum $\cdot$ J. W. Groothoff • J. C. M. Oostveen • \\ F. G. J. Oosterveld • C. P. van der Schans $\cdot$ R. Soer $\cdot$ M. F. Reneman
}

Received: 29 October 2009/ Accepted: 4 May 2010/Published online: 21 May 2010

(C) The Author(s) 2010. This article is published with open access at Springerlink.com

\begin{abstract}
Objective The prevalence of osteoarthritis (OA) increases, but the impact of the disorder on peoples' functional capacity is not known. Therefore, the objective of this study was to compare self-reported health status and functional capacity of subjects with early OA of hip and/or knee to reference data of healthy working subjects and to assess whether this capacity is sufficient to meet physical job demands.

Methods Self-reported health status and functional capacity of 93 subjects from the Cohort Hip and Cohort
\end{abstract}

H. J. Bieleman $(\bowtie)$ · F. G. J. Oosterveld

Expertise Center Health, Social Care and Technology,

Saxion University of Applied Sciences, P.O. Box 70.000,

$7500 \mathrm{~KB}$ Enschede, The Netherlands

e-mail: h.j.bieleman@saxion.nl

H. J. Bieleman - M. W. van Ittersum - J. W. Groothoff .

R. Soer - M. F. Reneman

Graduate School for Health Research, University Medical Center

Groningen, University of Groningen,

Groningen, The Netherlands

H. J. Bieleman · M. W. van Ittersum · J. W. Groothoff Department of Health Sciences, University Medical Center Groningen, University of Groningen,

Groningen, The Netherlands

M. W. van Ittersum - C. P. van der Schans

Hanze University of Applied Sciences, Groningen,

The Netherlands

J. C. M. Oostveen

Rheumatology Twente, Department of Rheumatology,

Ziekenhuisgroep Twente, Almelo, The Netherlands

R. Soer - M. F. Reneman

Center for Rehabilitation, University Medical Centre Groningen,

University of Groningen, Groningen, The Netherlands
Knee (CHECK) were measured using the Short-Form 36 Health Survey and 6 tests of the Work Well Systems Functional Capacity Evaluation. Results were compared with reference data from 275 healthy workers, using $t$-tests. To compare the functional capacity with job demands, the proportions of subjects with OA performing lower than the $\mathrm{p}_{5}$ of reference data were calculated.

Results Compared to healthy workers, the subjects (mean age 56) from CHECK at baseline reported a significantly worse physical health status, whereas the women $(n=78)$ also reported a worse mental health status. On the FCE female OA subjects performed significantly lower than their healthy working counterparts on all 6 tests. Male OA subjects performed lower than male workers on 3 tests. A substantial proportion of women demonstrated functional capacities that could be considered insufficient to perform jobs with low physical demands.

Conclusions Functional capacity and self-reported health of subjects with early OA of the hips and knees were worse compared to healthy ageing workers. A substantial proportion of female subjects did not meet physical job demands.

Keywords Osteoarthritis - Functional capacity · SF-36 $\cdot$ Job demands

\section{Introduction}

An increase in the participation in paid work of people in the age of 45-65 is considered necessary to afford the costs that are generated by the ageing of the population (Gobelet et al. 2007; Ilmarinen 2001; European Commission 2004). However, current knowledge about the health status and the functional capacity (the ability to perform work-related activities) of this worker category (Kenny et al. 2008; 
Berg van den et al. 2009; Broersen et al. 1996) raises the question whether this pursuit is realistic. Older workers with chronic diseases or disorders are specifically at risk of developing work disabilities and loosing their job (Kenny et al. 2008; Schuring et al. 2007). Regarding rheumatic diseases ample evidence indicates that rheumatoid arthritis (RA) has a negative impact on the work participation of patients (Zirkzee et al. 2008; Chorus et al. 2000). For osteoarthritis (OA), however, there is limited information with regard to work participation (Gobelet et al. 2007; Merx et al. 2007) and functional capacity for work-related activities (Bieleman et al. 2007). This disorder is of particular interest because of its increasing prevalence, related to the ageing of populations and the rising prevalence of overweight and obesity (Issa and Sharma 2006). Since people with OA often experience limitations in physical functioning, an effect on work participation may be anticipated. There is a lack of knowledge about the work status and functional capacity of people with early OA compared to healthy people. As a consequence, the need for (preventive) interventions to maintain functional capacity and to stimulate work participation remains unclear.

Several work-related and individual factors are related to work ability (Berg van den et al. 2009). One of the individual factors is the functional capacity, which can be assessed with a Functional Capacity Evaluation (FCE). An FCE is an evaluation of the capacity to perform activities that is used to make recommendations for participation in work, while considering the person's body functions and structures, environmental factors, personal factors and health status (Soer et al. 2009). FCE's are used in many countries worldwide in rehabilitation, occupational health care and insurance settings. Performance-based data provide clinicians with additional information about functioning that would be missed when relied on self-reports only (Reneman et al. 2002).

The aims of this paper were to assess the self-reported health status and the observed functional capacity of people with early OA in hips and/or knees and to compare these to a reference sample of healthy workers, matched for age and controlled for sex. It was assumed that the functional capacity of healthy workers was sufficient to meet the physical demands in their jobs. This comparison, therefore, enabled assessment of the functional capacity of subjects with $\mathrm{OA}$ in relation to physical job demands.

Research questions were:

1. Is the self-reported health status of subjects with early OA different from healthy workers?

2. Is the observed functional capacity of subjects with early OA different from healthy workers?

3. Is the functional capacity of subjects with early OA sufficient to meet physical job demands?

\section{Methods}

Design

Self-reported health status and functional capacity of a subsample from the Cohort Hip and Cohort Knee (CHECK) study on early osteoarthritis (Wesseling et al. 2009) were measured at baseline of this 10-year cohort study. Results on both measures were compared to reference data from a separate study that was performed in 702 healthy workers, with the aim to establish normative data (Soer et al. 2009).

\section{Subjects}

Inclusion criteria for the CHECK cohort were hip and/or knee complaints for which the subject visited the general practitioner no longer than 6 months ago and that were not attributed to direct trauma or other disorders. The age of the subjects at baseline was between 45 and 65 years. Exclusion criteria were the presence of inflammatory rheumatic disorders, joint prosthesis (hip and knee), previous joint trauma and serious co morbidity. Wesseling et al. (2009) concluded that subject characteristics $(n=1,002)$ at inclusion indeed label CHECK as an early OA cohort. Based on the classification by the Kellgren and Lawrence (1957) rating score, the proportion of subjects with radiological osteoarthritis ( $\mathrm{K}$ and $\mathrm{L}>1$ ) was $6 \%$ for the knee and $10 \%$ for the hip. However, $76 \%$ of the patients with knee symptoms could be diagnosed as OA according to the clinical ACR criteria for classification of knee OA (Altman et al. 1986). Only a minority of CHECK participants with hip symptoms (24\%) fulfilled the clinical classification criteria of hip OA (Altman et al. 1991). All participants provided written informed consent before entering the study, and the Medical Ethical Board of hospital 'Medisch Spectrum Twente' in Enschede, the Netherlands, approved the study.

In the healthy worker study (Soer et al. 2009), subjects between 20 and 61 years were included that were working in a wide range of professions and who reported no absenteeism due to musculoskeletal complaints in the year before the assessment. For this comparative study, the data from all subjects aged 45-61 were used (183 men and 92 women).

Measurements

\section{Self-reported health status}

All subjects filled out the Short-Form 36 Health Survey (SF-36, McHorney et al. 1993)). The SF-36 consists of 36 items that cover 8 aspects of health. The physical function, physical role, bodily pain and general health subscales 
together comprise the 'physical component' of the person's health status. The social function, emotional role, mental health and vitality subscales comprise the 'mental component' of a person's health status. All raw scores were transformed into scores in a range between 0 and 100 and a higher score on the subscales and components represented a better health status.

\section{Functional capacity}

The WorkWell Systems Functional Capacity Evaluation (Work Well Systems 2006) was used to assess subjects' capacity to perform work-related activities. Twenty-two tests, including all those that cause load bearing to the hips and the knees, were selected from the standardized 2-day WWS FCE protocol. These tests aim to record capacity with regard to manual material handling, working postures and movements and refer to physical strength, endurance or speed. Providing the evaluator judged the tests to be performed safely, based on observation criteria as movement pattern and postural changes (Reneman et al. 2002), subjects were asked to continue to a higher load level (5 repetitions per level). The static endurance tests were continued until a preset limit (15 min) was reached. The subject was free to end any test at any moment, for example because of discomfort or pain. Comparisons with the healthy workers were made on 6 standardized tests that represent physical job demands and that were performed in both populations. These tests, the reliability of which has been established (Gross and Battié 2002; Brouwer et al. 2003; Reneman et al. 2004; Soer et al. 2006; van Ittersum et al. 2009), are listed in the following paragraphs.

\section{Material Handling}

Lifting Low Objective: capacity of lifting from table to floor. Materials: plastic receptacle $(40 \times 30 \times 26 \mathrm{~cm})$, a wall-mounted system with adjustable shelves and weights of 1.0, 2.0 and $4.0 \mathrm{~kg}$. Procedure: five lifts from table at $74 \mathrm{~cm}$ to floor and vice versa in standing position within $90 \mathrm{~s}$. Four to five weight increments until maximum amount of $\mathrm{kg}$ was reached.

Overhead Lifting Objective: capacity of overhead lifting task. Materials: plastic receptacle $(40 \times 30 \times 26 \mathrm{~cm})$, a wall-mounted system with adjustable shelves and weights of $1.0,2.0$ and $4.0 \mathrm{~kg}$. Procedure: five lifts from table $(74 \mathrm{~cm})$ to crown height and vice versa in standing position within $90 \mathrm{~s}$. Four to five weight increments until maximum amount of $\mathrm{kg}$ was reached.

Carrying Objective: capacity of two handed carrying. Materials: plastic receptacle $(40 \times 30 \times 26 \mathrm{~cm})$, a wallmounted system with adjustable shelves and weights of 1.0 ,
2.0 and $4.0 \mathrm{~kg}$. Procedure: $20 \mathrm{~m}$ carrying at waist height with receptacle within $90 \mathrm{~s}$. Four to five weight increments until maximum amount of $\mathrm{kg}$ was reached.

\section{Postural tolerance}

Overhead Working Objective: capacity of postural tolerance of overhead working. Materials: aluminium plate adjustable in height with 20 holes, bolts and nuts and two cuff weights of $1.0 \mathrm{~kg}$ each. Procedure: standing with hands at crown height, manipulating nuts and bolts wearing cuff weights around the wrists. The time that position is held was measured (seconds).

\section{Coordination and repetitive movements}

Dynamic Bending Objective: capacity of repetitive bending and reaching. Materials: 20 marbles and 2 bowls with a $14 \mathrm{~cm}$ diameter positioned at floor and crown height. Procedure: standing with knees flexed between 0 and $30^{\circ}$, move marbles vertically from floor to crown height as fast as possible. Time needed to remove 20 marbles is scored (seconds).

Repetitive Side Reaching Objective: capacity of fast repetitive side movements of the upper extremity. Materials: 30 marbles and 2 bowls with a $14 \mathrm{~cm}$ diameter positioned at table height $(74 \mathrm{~cm})$. Procedure: sitting with bowls on wingspan distance, move marbles horizontally at table height from right to left with right arm as fast as possible and vice versa. Time needed to move 30 marbles is scored (seconds).

Preceding the FCE tests subjects' age and sex were registered. Length and weight measurements were performed to calculate Body Mass Index (BMI). Tests were administered by 4 th year physical therapy students who had received one-day training in the procedures and the execution of the FCE. They were trained and supervised by the research team.

\section{Statistical analysis}

Reference data were matched for age and controlled for sex. For FCE results, two age categories were distinguished to allow analysis of the influence of ageing. Because of the small number of male subjects, the data were also compared for the whole group, to increase the statistical power. To answer study questions 1 and 2, SF36 scores and FCE results of subjects with early OA and of the healthy workers were compared using $t$-tests. Mean differences and 95\% confidence intervals between the groups were analysed. 
Table 1 Subject characteristics

\begin{tabular}{|c|c|c|c|c|c|c|}
\hline \multirow[b]{2}{*}{ Variable } & \multicolumn{3}{|l|}{ Males } & \multicolumn{3}{|l|}{ Females } \\
\hline & Early OA & Healthy & Mean difference $(95 \% \mathrm{CI})$ & Early OA & Healthy & Mean difference $(95 \% \mathrm{CI})$ \\
\hline$n$ & 15 & 183 & & 78 & 92 & \\
\hline Paid job (\%) & 47 & 100 & & 47 & 100 & \\
\hline \multicolumn{7}{|l|}{ Age in years: } \\
\hline Mean (SD) & $58(5.3)$ & $52(4.1)$ & $-6(-8.2--3.8)^{*}$ & $56(4.8)$ & $52(4.0)$ & $-4(-5.3--2.7)^{*}$ \\
\hline Range & $48-65$ & $46-61$ & & $48-66$ & $46-59$ & \\
\hline Body mass index\# & $25.8(5.3)$ & $25.6(3.9)$ & $-0.2(-1.9-2.3)$ & $26.2(4.3)$ & $24.1(3.1)$ & $-2.1(-3.2--0.9)^{*}$ \\
\hline \multicolumn{7}{|l|}{ SF-36\# } \\
\hline Physical function & $80.5(8.2)$ & $96.6(5.7)$ & $16.1(12.9-19.3)^{*}$ & $69.8(22.8)$ & $94.7(8.1)$ & $24.9(19.8-30.0)^{*}$ \\
\hline Physical role & $80.4(32.8)$ & $93.1(19.2)$ & $12.7(1.3-24.1)^{*}$ & $56.6(43.5)$ & $93.4(19.6)$ & $36.8(26.4-47.2)^{*}$ \\
\hline Bodily pain & $71.9(12.8)$ & $90.3(12.7)$ & $18.4(11.5-25.3)^{*}$ & $64.3(19.1)$ & $92.1(9.9)$ & $27.8(23.2-32.4)^{*}$ \\
\hline General health & $48.2(18.3)$ & $75.0(13.7)$ & $26.8(19.2-34.4)^{*}$ & $52.6(18.7)$ & $76.7(15.0)$ & $24.1(18.4-29.8)^{*}$ \\
\hline Social function & $92.0(11.6)$ & $91.3(13.2)$ & $-0.70(-7.8-6.4)$ & $74.5(20.4)$ & $90.6(11.8)$ & $16.1(11.0-21.2)^{*}$ \\
\hline Emotional role & $95.2(17.8)$ & $96.7(15.3)$ & $1.5(-6.9-9.9)$ & $82.0(32.9)$ & $91.8(23.5)$ & $9.8(1.0-18.6)^{*}$ \\
\hline Mental health & $80.6(11.3)$ & $72.4(10.2)$ & $-8.2(-13.8--2.6)^{*}$ & 73.7 (13.7) & $71.0(9.0)$ & $-2.7(-6.3-0.9)$ \\
\hline Vitality & $66.4(13.2)$ & $69.1(11.5)$ & $2.7(-3.6-9.0)$ & $59.8(16.6)$ & $66.0(13.0)$ & $6.2(1.6-10.8)^{*}$ \\
\hline
\end{tabular}

Differences between early OA (CHECK) and healthy workers

$* p<0.05$; \# mean (SD)

\section{Use of the 5th percentile as reference for job demands}

The rationale behind the study question about job demands is that the reference data were established to assist clinicians in assessing the functional capacity of a patient. By comparison with the reference values, a patient's capacity can be classified into a physical demand category (sedentary-light - medium-heavy — very heavy) according to the Dictionary of Occupational Titles (DOT, U.S. Department of Labor 1991). It was assumed that the functional capacity of healthy workers was at least equal to their workload, because they worked $20 \mathrm{~h}$ or more per week, with no absenteeism due to musculoskeletal complaints during 1 year before the FCE. Therefore, this capacity may be considered the 'norm' to which the functional capacity of patients can be compared. We chose to compare the results of the subjects with $\mathrm{OA}$ to the 5th percentile scores of the reference data on the lowest category, DOT-1 ('sedentary work', with occasionally lifting up to $4.5 \mathrm{~kg}$ ): if the relatively weakest of the healthy workers can still meet their job demands, their functional capacity may be used as reference point.

\section{Results}

\section{Subjects}

Subject characteristics and self-reported health status are presented in Table 1. Compared to healthy workers, subjects with early OA were older and less than half of them had a paid job. Women with early OA had a statistically significantly higher BMI than the female healthy workers.

Health status comparison

The subjects with OA reported statistically significantly lower scores than the healthy workers on the physical component of SF-36, for both sexes. On the mental component, the CHECK women also scored statistically significantly lower than the healthy subjects, with exception of the mental health scale. The scores on the mental component of SF-36 for the male healthy workers and the men with OA were similar, but on the mental health subscale, the men with OA scored significantly higher than the healthy working men. Because of the higher mean age and the small number of the male subjects with OA, afterwards a corrected analysis was performed, in which they were compared to an age-matched subsample of 30 healthy workers (mean age 58). This analysis generated similar results on all scales (not presented here). The healthy working men and women had very similar scores, whereas in the OA subjects, the men scored higher than the women.

Functional capacity comparison

The FCE test results for the male subjects are presented for separate age categories and for the total group (Table 2). 
Table 2 FCE performances of male subjects with early OA (CHECK, $n=15)$ and male healthy workers $(n=183)$

\begin{tabular}{lllll}
\hline FCE test & $\begin{array}{l}\text { Age category \# } \\
\text { (years) }\end{array}$ & $\begin{array}{l}\text { Early OA } \\
\text { mean (SD) }\end{array}$ & $\begin{array}{l}\text { Healthy workers } \\
\text { mean (SD) }\end{array}$ & $\begin{array}{l}\text { Mean difference healthy-early } \\
\text { OA (95\% CI) }\end{array}$ \\
\hline Lifting low (kg) & $45-54$ & $31.8(7.4)$ & $44.9(12.3)$ & $13.2(1.0-25.4)^{*}$ \\
& $55-65$ & $34.1(6.1)$ & $43.0(14.5)$ & $9.0(3.5-14.4)^{*}$ \\
& All & $33.5(6.3)$ & $44.3(13.0)$ & $10.9(7.0-14.8)^{*}$ \\
Lifting Overhead (kg) & $45-54$ & $19.8(2.9)$ & $20.1(4.8)$ & $0.4(-4.4-5.2)$ \\
& $55-65$ & $17.3(3.9)$ & $18.9(4.6)$ & $1.6(-1.4-4.5)$ \\
& All & $17.9(3.7)$ & $19.7(4.8)$ & $0.1(-0.7-4.3)$ \\
Carry 2 hand (kg) & $45-54$ & $46.3(13.4)$ & $46.4(11.0)$ & $7.4(-0.9-15.7)$ \\
& $55-65$ & $35.7(11.5)$ & $43.1(12.7)$ & $7.0(0.7-13.1)^{*}$ \\
& All & $38.5(12.5)$ & $45.4(11.7)$ & $33(-93-160)$ \\
Overhead work (s) & $45-54$ & $236(103)$ & $269(127)$ & $63(-0.4-127.1)$ \\
& $55-65$ & $207(61)$ & $270(102)$ & $55(-7-117)$ \\
Dynamic bend (s) & All & $214(72)$ & $270(119)$ & $-4(-11-3)$ \\
& $45-54$ & $51(7)$ & $47(6)$ & $4(-74-82)$ \\
& $55-65$ & $62(16)$ & $66(128)$ & $-12(3-21)^{*}$ \\
Rep. side reach (s) & All & $60(15)$ & $48(7)$ & $4(-11-19)$ \\
& $45-54$ & $76(17)$ & $80(12)$ & $-15(-30-0.0)$ \\
& $55-65$ & $95(20)$ & $80(11)$ & $-11(-23-2)$ \\
\hline
\end{tabular}

\# CHECK: 45-54: $n=4,55-65: n=11$, All: $n=15$; Healthy: 45-54: $n=128,55-60: n=55$, All: $n=183$

* significant at alpha $=0.05$

The capacity for 'lifting low' was significantly lower in the CHECK men from both age-groups compared to the healthy workers. The other tests showed no significant differences between the subjects with OA and the reference data in the age categories. For the comparisons between the total groups, the differences in the tests lifting low, carrying-2-handed and dynamic bending were significant; the healthy workers lifted and carried more weight and were faster on dynamic bending.

In Table 3, the FCE test results for the female subjects are presented.

The female subjects with OA performed significantly lower than the female healthy working subjects on all tests. In both groups, the younger subjects performed higher than the older; the differences were larger in the OA subjects.

Functional capacity versus physical job demands

To assess whether the functional capacity of subjects with early OA was sufficient to meet the physical job demands, the results were compared to the fifth percentile of the results of the healthy workers. In Table 4 , these $p_{5}$ scores are presented, followed by the proportion of subjects with OA that performed below this cut-off value.

The men with early OA all scored above p5, except on the dynamic bending test. One of the older men scored below p5 on the overhead working posture test. On all tests, $20-40 \%$ of the younger women and $25-65 \%$ of the older women scored below p5.

\section{Discussion}

This study revealed that both the 15 male and the 78 female subjects from a subsample from the CHECK cohort at baseline reported a worse physical health status (SF-36) compared to the healthy ageing workers, whereas the women also reported a worse mental health status on 3 out of 4 scales. On the FCE, the female CHECK subjects performed significantly lower than their healthy working counterparts on all 6 tests. The male subjects with OA performed lower on 3 out of 6 tests. A substantial proportion of female subjects demonstrated functional capacities that would be considered insufficient to meet the lowest category of physical job demands.

The worse physical health status as reported on the SF-36 can be attributed to the knee or hip complaints of the subjects, but other physical factors may also have influenced their health status. Serious comorbidity was an exclusion criterion for the CHECK cohort, but back pain and other musculoskeletal discomfort were frequently reported. Contrarily, an over representation of physically strong and healthy volunteers in the reference population may have introduced bias that explains part of the observed 
Table 3 FCE test performances of female subjects with early OA (CHECK, $n=78)$ and female healthy workers $(n=92)$

\begin{tabular}{|c|c|c|c|c|}
\hline FCE test & $\begin{array}{l}\text { Age category \# } \\
\text { (years) }\end{array}$ & $\begin{array}{l}\text { Early OA } \\
\text { mean }(\mathrm{SD})\end{array}$ & $\begin{array}{l}\text { Healthy workers } \\
\text { mean (SD) }\end{array}$ & $\begin{array}{l}\text { Mean difference } \\
\text { healthy-early OA }(95 \% \mathrm{CI})\end{array}$ \\
\hline \multirow[t]{3}{*}{ Lifting Low (kg) } & $45-54$ & $19.0(6.9)$ & $25.7(8.7)$ & $6.7(3.3-10.1)^{*}$ \\
\hline & $55-65$ & $15.5(6.8)$ & $23.6(7.3)$ & $8.1(4.5-11.6)^{*}$ \\
\hline & All & $17.0(7.0)$ & $24.8(8.5)$ & $7.8(5.3-10.2)^{*}$ \\
\hline \multirow[t]{3}{*}{ Lifting overhead $(\mathrm{kg})$} & $45-54$ & $9.2(3.8)$ & $11.5(3.4)$ & $2.3(0.8-3.8)^{*}$ \\
\hline & $55-65$ & $7.0(3.1)$ & $10.5(3.3)$ & $3.5(1.9-5.1)^{*}$ \\
\hline & All & $8.0(3.6)$ & $11.2(3.3)$ & $3.2(2.1-4.2)^{*}$ \\
\hline \multirow[t]{3}{*}{ Carry 2 hand (kg) } & $45-54$ & $22.1(5.6)$ & $28.3(7.5)$ & $6.2(3.3-9.0)^{*}$ \\
\hline & $55-65$ & $17.1(6.4)$ & $26.6(8.0)$ & $9.5(6.0-13.1)^{*}$ \\
\hline & All & $19.3(6.5)$ & $27.7(7.7)$ & $8.3(6.1-10.5)^{*}$ \\
\hline \multirow[t]{3}{*}{ Overhead work (s) } & $45-54$ & $163(67.8)$ & $239(111)$ & $77(42-112)^{*}$ \\
\hline & $55-65$ & $157(79.4)$ & $234(75)$ & $76(36-117)^{*}$ \\
\hline & All & $160(74)$ & $233(103)$ & $73(45-101)^{*}$ \\
\hline \multirow[t]{3}{*}{ Dynamic bend (s) } & $45-54$ & $55(16.0)$ & $45(5.6)$ & $-10(-16--4)^{*}$ \\
\hline & $55-65$ & $64(15.2)$ & $46(7.1)$ & $-18(-24--13)^{*}$ \\
\hline & All & $60(16)$ & $45(6)$ & $-15(-19--11)^{*}$ \\
\hline \multirow[t]{3}{*}{ Rep. side reach (s) } & $45-54$ & $84(25.8)$ & $74(9.1)$ & $-10(-19-0.0)^{*}$ \\
\hline & $55-65$ & $90(15.5)$ & $78(10.2)$ & $-13(-19--6)^{*}$ \\
\hline & All & $87(21)$ & $75(9)$ & $-12(-17--7)^{*}$ \\
\hline
\end{tabular}

\# CHECK: 45-54: $n=34,55-65: n=43$, All: $n=77$; Healthy: 45-54: $n=68,55-60: n=24$, All: $n=92$

* significant at alpha $=0.05$

Table 4 Proportions of subjects with early $\mathrm{OA}$ (CHECK) performing below $(<)$ fifth percentile $\left(\mathrm{p}_{5}\right)$ of reference data of healthy workers

\begin{tabular}{lllll}
\hline FCE test: & & $\mathrm{p}_{5}$ score: (DOT-1) & $\begin{array}{l}\% \text { males scoring } \\
<\mathrm{p}_{5}(n=15)\end{array}$ & $\begin{array}{l}\% \text { females scoring } \\
<\mathrm{p}_{5}(n=78)\end{array}$ \\
\hline Lifting low & $45-54$ & $16 \mathrm{~kg}$ & 0 & 35 \\
& $55-65$ & & 0 & 55 \\
Lifting high & $45-54$ & $7 \mathrm{~kg}$ & 0 & 33 \\
& $55-65$ & & 0 & 50 \\
Carrying & $45-54$ & \multirow{2}{*}{$16 \mathrm{~kg}$} & 0 & 20 \\
& $55-65$ & & 0 & 45 \\
Overhead Work & $45-54$ & $101 \mathrm{~s}$ & 0 & 20 \\
& $55-65$ & & 9 & 25 \\
Dynamic Bend & $45-54$ & $55 \mathrm{~s}$ & 33 & 38 \\
& $55-65$ & & 45 & 65 \\
Rep. Side Reach & $45-54$ & $93 \mathrm{~s}$ & 0 & 22 \\
& $55-65$ & & 0 & 40 \\
\hline
\end{tabular}

differences. Still, the early phase of OA is clearly accompanied by self-reported limitations in physical function and physical roles for both sexes and also by mental health limitations for women.

The worse self-reported health status of the subjects with early OA compared to the healthy working subjects was also reflected in a lower functional capacity as measured on the FCE. The pain and stiffness in the hips or knees, possibly in combination with other health complaints, seem to have affected their performance in work- related physical activities. We reported earlier that in this sample the subjects with low self-reported functional status showed lower performances on the FCE (Bieleman et al. 2009).

About half of the subjects with early OA in this study did not have a paid job. Either or not having a paid job has been reported to explain part of the performance on an FCE (Bieleman et al. 2007). For example, on 'lifting low' the average difference between women from this study with paid work and those without paid work was $4.7 \mathrm{~kg}$ 
$(19.4 \mathrm{~kg}$ vs. $14.7 \mathrm{~kg})$. However, after correcting for this factor, there still remains a substantial difference between the capacities of the working subjects with early OA and the reference group of healthy workers. Therefore, it was concluded that in the early phase of OA of the hips and knees a decreased functional capacity is seen, both in working people and even more in people without paid work. The impact of the OA, as measured by self-reports and an FCE and compared to healthy workers, seems to be stronger in women than in men, both physically and mentally. Mental health factors may be related to having a job, either because a job requires for example vitality, or because of the social relations that a job may offer. Since many women in the study never had a job, this may explain the differences with the men.

The basis assumption for clinical interpretation of the results was that the functional capacity of healthy workers, used as reference data in this study, is equal to or exceeding their workload. For this reason, these data may be considered the "norm" to which the functional capacity of the subjects with OA could be compared (Soer et al. 2009). To be precise, the $\mathrm{p}_{5}$ scores of the reference data for working subjects with the physically least demanding jobs (DOT-1; sedentary work) were used as reference. A substantial proportion of the female CHECK subjects performed lower than this $\mathrm{p}_{5}$ score. For the persons with paid work amongst them, the low performance indicated that they could be considered to be at risk of not meeting their physical work load. For those without paid work, a low functional capacity might impair their physical activities of daily living (ADL) and leisure. The influence of OA on role participation has been identified as an important research issue (Gignac et al. 2008; Hunt et al. 2008). The subjects without paid work formed the majority of the group who performed lower than $\mathrm{p}_{5}$, which is consistent with the earlier discussion on the relation between having paid work and FCE performance.

It may be argued that only patients with OA who are physically functioning relatively well are able to perform paid work and to live an active lifestyle in ADL and leisure. However, work and an active lifestyle can also be postulated to have beneficial effects on physical functioning and health. Physical activity in Japanese women with hip OA was related to both work status and to the degree of OA, but only the women without paid work were physically inactive, whereas the workers were not (Hirata et al. 2006). The hypothesis of a physically conditioning effect of work and an interaction with life-style seems to be supported by other observations in our study. The female healthy workers had a significantly lower BMI than the women with early OA (24.1 vs. 26.2). The smaller impact of early OA on health and functional status in men compared to women could also illustrate the conditioning effect of work. The men without paid work only recently retired and may still have had the conditioning benefit of their past working life, whereas many of the women reported never to have had paid work. Furthermore, the women also performed lower on FCE tests that do not relate to knee or hip function, such as working overhead. Yet, considering the cross-sectional nature of our study and the small number of male subjects, full explanations for these observations cannot be given. The relations between work, health status and functional capacity should be studied longitudinally.

Another limitation of the study is that no more than 6 tests in our protocol matched those from the reference study. However, these tests cover the aspects of strength, static endurance and speed/mobility. Together, this should provide a valid impression of the ability to perform workrelated activities, relevant for people with early OA. The validity of shorter FCE protocols, which obviously have practical advantages, has been demonstrated in a recent study (Gross et al. 2007). Several alternative explanations besides the OA may theoretically explain parts of the differences in results between the groups, as for example testing order and fatigue, age, and willingness to give maximal effort. Considering age, the CHECK subjects were up to 65 years old whereas the oldest working subjects were 61. Soer et al. (2009) constructed a regression model for predicting the result on 'lifting low' in which the coefficient for age was $-0.2 \mathrm{~kg} /$ year. Applying this value to the difference in mean age between our groups ( 6 years for men, 4 years for women) would generate an expected difference of 1.2 and $0.8 \mathrm{~kg}$, respectively. Clearly, the differences we found were much larger than could be expected only on the basis of the age difference. Hence, it appears that the functional limitations of the subjects with early OA should actually be attributed to the observed lower capacity that accompanied their complaints.

Functional capacity is one of the several components that determine work ability and social participation (Berg van den et al. 2009; Hunt et al. 2008). Experts in the field of disability claims and return to work have different opinions on the utility of FCE (Wind et al. 2006), but FCE information had complementary value according to most insurance physicians (Wind et al. 2009). Our study indicates a potential preventive use of FCE. The results demonstrated that less than half of the subjects with early OA had paid work and that both their self-reported health status and their functional capacity were significantly lower compared to healthy working subjects. A substantial proportion of women did not meet the physical job demands. Therefore, considering the aim to increase the work participation (preventive) interventions would be needed. For the workers amongst our subjects, adapting the working situation and maintaining functional capacity is recommendable. For others who consider finding a job (again), 
increasing their functional capacity and selecting jobs without heavy physical demands is advisable to facilitate actual work participation.

Acknowledgments "CHECK is funded by the Dutch Arthritis Association on the lead of a steering committee comprising 16 members with expertise in different fields of OA chaired by Prof. J.W.J. Bijlsma and coordinated by J. Wesseling, MSc. Involved are: Academic Hospital Maastricht; Erasmus Medical Center Rotterdam; Jan van Breemen Institute/VU Medical Center Amsterdam; Kennemer Gasthuis Haarlem; Martini Hospital Groningen/Allied Health Care Center for Rheumatology and Rehabilitation Groningen; Medical Spectrum Twente Enschede/Twenteborg Hospital Almelo; St. Maartenskliniek Nijmegen; Leiden University Medical Center; University Medical Center Utrecht and Wilhelmina Hospital Assen." Grants for this study were received from NutsOhra Fonds and Mobiliteitsfonds HBO. The authors are grateful to the subjects who actively participated in the study and to the students that led the FCE tests. Also they thank Anita Mooij and Annet ter Avest, research nurses of the Medisch Spectrum Twente and the Ziekenhuisgroep Twente, Wim Hilberdink, physical therapist in Groningen, and Janet Wesseling, CHECK-coordinator, for their contributions to the study.

Conflict of interest statement The authors declare that they have no conflict of interest.

Open Access This article is distributed under the terms of the Creative Commons Attribution Noncommercial License which permits any noncommercial use, distribution, and reproduction in any medium, provided the original author(s) and source are credited.

\section{References}

Altman R, Asch E, Bloch D, Bole G, Borenstein D, Brandt K et al (1986) Development of criteria for the classification and reporting of osteoarthritis. Classification of osteoarthritis of the knee. Diagnostic and therapeutic criteria committee of the american rheumatism association. Arthritis Rheum 29:1039-1049

Altman R, Alarcon G, Appelrouth D, Bloch D, Borenstein D, Brandt $\mathrm{K}$ et al (1991) The American college of rheumatology criteria for the classification and reporting of osteoarthritis of the hip. Arthritis Rheum 34:505-514

Berg van den TIJ, Elders LAM, de Zwart BCH, Burdorf A (2009) The effects of work-related and individual factors on the work ability index: a systematic review. Occup Environ Med 66:211-220

Bieleman HJ, van Ittersum MW, Groothoff JW, Reneman MF, van der Schans CP, Oosterveld FGJ (2007) Arbeidsbelastbaarheid van mensen met beginnende heup- en knieklachten. Een verkennend onderzoek in het CHECK artrosecohort. Work capacity of people with early complaints of hip and knee. An explorative study in the CHECK osteoarthritis cohort. Ned T Fysiotherapie 117(6):225-232

Bieleman HJ, Reneman MF, van Ittersum MW, van der Schans CP, Groothoff JW, Oosterveld FGJ (2009) Self-reported functional status as predictor of observed functional capacity in subjects with early osteoarthritis of the hip and knee. A diagnostic study in the CHECK cohort. J Occup Rehabil 19(4):345-353

Broersen JP, de Zwart BC, van Dijk FJ, Meijman TF, van Veldhoven M (1996) Health complaints and working conditions experienced in relation to work and age. Occup Environ Med 53(1):51-57

Brouwer S, Reneman MF, Dijkstra PU, Groothoff JW, Schellekens JM, Goeken LN (2003) Test-retest reliability of the Isernhagen work systems functional capacity evaluation in patients with chronic low back pain. J Occup Rehabil 13(4):207-218

Chorus AMJ, Miedema HS, Wevers CJ, Van der Linden S (2000) Labour force participation among patients with rheumatoid arthritis. Ann Rheum Dis 59:549-554

European Commission, report COM (2004) 146, "Increasing employment of older workers and delaying the exit from the labour market"

Gignac MAM, Backman CL, Davis AM, Lacaille D, Mattison CA, Montie $\mathrm{P}$ et al (2008) Understanding social role participation: what matters to people with arthritis? J Rheum 35(8):1655-1663

Gobelet C, Luthi F, Al-Khodairy AT, Chamberlain MA (2007) Work in inflammatory and degenerative joint diseases. Disabil Rehabil 29(17):1331-1339

Gross DP, Battié M (2002) Reliability of safe maximum lifting determinations of a functional capacity evaluation. Phys Ther 82(4):364-371

Gross DP, Battié MC, Asante AK (2007) Evaluation of a short-form functional capacity evaluation: less may be best. J Occup Rehab 3(17):422-435

Hirata S, Ono R, Yamada M, Takikawa S, Nishiyama T, Hasuda K et al (2006) Ambulatory physical activity, disease severity, and employment status in adult women with osteoarthritis of the hip. J Rheumatol 33:939-945

Hunt MA, Birmingham TB, Skarakis-Doyle E, Vandervoort AA (2008) Towards a biopsychosocial framework of osteoarthritis of the knee. Disabil Rehabil 30(1):54-61

Ilmarinen JE (2001) Aging workers. Occup Environ Med 58:546-552

Issa SN, Sharma L (2006) Epidemiology of osteoarthritis: an update. Curr Rheumatol Rep 8(1):7-15

Ittersum MW, Bieleman HJ, Reneman MF, Oosterveld FGJ, Groothoff JW, van der Schans CP (2009) Functional capacity evaluation in subjects with early osteoarthritis of hip and/or knee; is two- day testing needed? J Occup Rehabil 19(3):238-244

Kellgren JH, Lawrence JS (1957) Radiological assessment of osteoarthrosis. Ann Rheum Dis 16:494-502

Kenny GP, Yardley JE, Martineau L, Jay O (2008) Physical work capacity in older adults: implications for the aging worker. Am J Ind Med 51(8):610-625

McHorney CA, Ware JE, Racze AE (1993) The MOS 36 item shortform health status survey (SF-36): II. Psychometric and clinical tests of validity in measuring physical and mental health constructs. Med Care 31((3):247-263

Merx H, Dreinhofer KE, Gunther KP (2007) Sozialmedizinische Bedeutung der Arthrose in Deutschland. Z Orthop Unfall 145:421-429

Reneman MF, Jaegers SM, Westmaas M, Goeken LN (2002) The reliability of determining effort level of lifting and carrying in a functional capacity evaluation. Work 18(1):23-27

Reneman MF, Brouwer S, Meinema A, Dijkstra PU, Geertzen JH, Groothoff JW (2004) Test-retest reliability of the Isernhagen work systems functional capacity evaluation in healthy adults. J Occup Rehabil 14(4):295-305

Schuring M, Burdorf L, Kunst A, Mackenbach J (2007) The effects of ill health on entering and maintaining paid employment: evidence in European countries. J Epidemiol Community Health 61:597-604

Soer R, Gerrits EH, Reneman MF (2006) Test-retest reliability of a WRULD functional capacity evaluation in healthy adults. Work 26(3):273-280

Soer R, van der Schans CP, Geertzen JHB, Brouwer S, Dijkstra PU, Groothoff JW et al (2009) Normative values for a functional capacity evaluation. Arch Phys Med Rehabil 90(10):17851794

U.S. Department of Labor (1991) Employment and training administration dictionary of occupational titles, 4 th edn 
Wesseling J, Dekker J, Van den Berg WB, Bierma-Zeinstra SMA, Boers M, Cats HA et al (2009) CHECK: cohort hip \& cohort knee; similarities and differences with the OA initiative. Ann Rheum Dis 68(9):1413-1419

Wind H, Gouttebarge V, Kuijer PP, Sluiter JK, Frings-Dresen MH (2006) The utility of functional capacity evaluation: the opinion of physicians and other experts in the field of return to work and disability claims. Int Arch Occup Environ Health 79(6):528-534

Wind H, Gouttebarge V, Kuijer PP, Sluiter JK, Frings-Dresen MH (2009) Complementary value of functional capacity evaluation for physicians in assessing the physical work ability of workers with musculoskeletal disorders. Int Arch Occup Environ Health 82(4):435-443

WorkWell Systems (2006) Functional capacity evaluation V. 2. WorkWell Systems INC, Duluth, MN, USA

Zirkzee EJM, Sneep AC, de Buck PDM, Allaart CF, Peeters AJ, Ronda KH et al (2008) Sick leave and work disability in patients with early arthritis. Clin Rheumatol 27:11-19 